\title{
The Prime Cause and Treatment of Cancer
}

Niknamian $\mathrm{S}^{1 *}$ and Zaminpira $\mathrm{S}^{2}$
Department of Medicine, Liberty University, USA

*Corresponding author: Sorush Niknamian, Department of Medicine, Liberty University,

USA, Email: So.niknamian@gmail.com

\section{Research Article \\ Volume 3 Issue 1}

Received Date: January 08, 2020

Published Date: February 03, 2020

DOI: $10.23880 /$ oajmms-16000115

\section{Abstract}

Introduction: Cancer disease has become the first cause of death in the United States and world-wide. Most Researchers estimate that 595,690 of American people will die from cancer at the end of the year 2017. That means 1,600 deaths/day approximately. Cancer in modern societies is commonly treated with the combination of organ surgery, chemotherapy and radiotherapy which has many side effects and the high possibility of metastasis. Many kinds of diet strategies have been experimented. However, none of them have been particularly effective.

Materials and Methods: According to Otto Warburg hypothesis in the 1930's, the cause of cancer is the change in the metabolism of mitochondrion in human cells. Low oxygen in tissues in combination with high blood glucose will change the cell respiration from aerobic to anaerobic which leads to fermentation type of respiration. Professor Thomas N Seyfried in 2012 proposed cancer as a metabolic-mitochondrial disease. He has mentioned that all cancer cells' mitochondria are damaged and do not work properly.

Results: Mitochondrion is a microorganism living inside our cells and produce energy in the form of Adenosine Triphosphate (ATP) from fats, glucose and glutamine. In order to produce ATP, mitochondria need oxygen through the krebs cycle which is called "Aerobic Respiration". On the other hand, when the amounts of oxygen go under $30 \%$ in the tissues and cells, mitochondria cannot respire properly which causes the increase in the amounts of Reactive Oxygen Species (ROS) or better called "Inflammation" inside the cells and causes damage to the mitochondria. According to Professor Thomas N Seyfried, the main cause of cancer is the increase in ROS inside normal cells [Thomas N Seyfried et al., 2012].

Conclusion: In 2017 we (MAJ Dr. Sorush Niknamian and Professor Dr. Somayeh Zaminpira along with the continuous help of Professor Seyfried and Professor Stephanie Seneff hypothesized with the sponsorship of the Weston A. Price Foundation (WAPF), that cancer is an Evolutionary Metabolic Disease which we proposed in the $4^{\text {th }}$ International Conference in Medicine and Neurological Diseases which held in Fulda/Germany.

Keywords: Cancer Treatment; Adenosine Triphosphate; Intravenous Ozone Therapy; Ketogenic Diet

Abbreviations: ATP: Adenosine Triphosphate; ROS: Reactive Oxygen Species; WAPF: Weston A Price Foundation; KD: Ketogenic Diet; VCI: Violet Cancer Institute; SCTP: Sorush Cancer Treatment Protocol; EMHC: Evolutionary Metabolic Hypothesis of Cancer; SKD: Specific Ketogenic Diet; IOT: Intravenous Ozone Therapy.

\section{Introduction}

\section{Evolutionary Metabolic Hypothesis of Cancer (EMHC)}

The first living cells on Earth are thought to have arisen more than $3.5 \times 109$ years ago, when the Earth was not more than about 109 years old. The environment lacked oxygen 


\section{Open Access Journal of Mycology \& Mycological Sciences}

but was presumably rich in geochemically produced organic molecules, and some of the earliest metabolic pathways for producing ATP may have resembled present-day forms of fermentation. In the process of fermentation, ATP is made by a phosphorylation event that harnesses the energy released when a hydrogen-rich organic molecule, such as glucose, is partly oxidized. The electrons lost from the oxidized organic molecules are transferred via NADH or NADPH to a different organic molecule or to a different part of the same molecule, which thereby becomes more reduced. At the end of the fermentation process, one or more of the organic molecules produced are excreted into the medium as metabolic waste products. Others, such as pyruvate, are retained by the cell for biosynthesis. The excreted end-products are different in different organisms, but they tend to be organic acids. Among the most important of such products in bacterial cells are lactic acid which also accumulates in anaerobic mammalian glycolysis, and formic, acetic, propionic, butyric, and succinic acids. The first cell on the earth before the entrance of the bacteria did contain nucleus and used the fermentation process to produce ATP for its energy. Then an aerobic proteo-bacterium enters the eukaryote either as a prey or a parasite and manages to avoid digestion. It then became an endosymbiont [1-5].

\section{Discussions and Results}

As we observe, the fermentation process used the glucose or even glutamine to produce ATP, but the aerobic process used the glucose, fat and protein to produce more ATP than the previous one. The symbio-genesis of the mitochondria is based on the natural selection of Charles Darwin. Based on Otto Warburg Hypothesis, in nearly all cancer cells, the mitochondrion is shut down or is defected and the cancer cell does not use its mitochondrion to produce ATP. This process of adaptation is based on Lamarckian Hypothesis of Evolution and the normal cells goes back to the most primitive time of evolution to protect itself from apoptosis and uses the fermentation process like the first living cells 1.5 billion years ago. Therefore, cancer is an evolutionary metabolic disease which uses Glucose and Glutamine as the main food to produce ATP and Lactic Acid.

In another study, we hypothesized that increasing the ROS in a cell can cause damage to the DNA of the mitochondrion and also Nucleus DNA, but another reason behind turning the normal cell into cancer cell is the chaos caused by the increasing of inflammation inside each cell and increasing the intracellular ROS. These chaoses cause some abnormal messaging between the DNA of the nucleus to stop the apoptosis and turning the oxidative phosphorylation to the fermentation in cytosol. Normally by damaging to the mitochondria, the cell should apoptosis. However; the nucleus sends wrong messages to stop the apoptosis and do fermentation process in cytosol to survive the cell. Even some normal left mitochondria would be shut down and stop the oxidative phosphorylation. This is the main and the real reason how increasing intracellular inflammation [5].

With all the information above, the basic way to stop respiration of cancer cells is to stop feeding them. The main source of fuel in cancer cells are Glucose and Glutamine. The basic diet for weakening or even killing these cells is Ketogenic Diet (KD).

Ketogenic diet is a kind of regime which uses high fat content and low carbohydrate. This diet changes the metabolic state into the condition called Ketosis. After several days, fat becomes your body's primary energy source which causes an increase in the levels of compounds which is called "ketones" in the blood [6]. In general, a ketogenic diet used for weight loss is about $60-75 \%$ of calories as fat, with $15-30 \%$ of calories from protein and $5-10 \%$ of calories from carbs. However, when a ketogenic diet is being used therapeutically for the treatment of cancer, the fat content may be significantly higher that is up to $90 \%$ of calories, and the protein content lower [7].

Presently, limited researches do seem to show that a ketogenic diet may reduce tumor size and rate of progression in certain cancers. One of the few documented published case studies was performed on a 65-year-old woman with brain cancer. Following surgery, she received a ketogenic diet. Meanwhile, the tumor's progression slowed. But, 10 weeks after returning to a normal diet, she experienced a significant increase in tumor growth [8]. Similar case reports examined the reactions to a ketogenic diet in two young girls who were undergoing treatment for advanced brain cancer. Researchers observed that glucose uptake was decreased in the tumors of both patients. One of the girls reported that her quality of life had improved and remained on the diet for 12 months. During that time her cancer showed no further progression [9]. One study monitored tumor growth in response to a high-carb versus a ketogenic diet in 27 patients with cancer of the digestive tract. Tumor growth increased by $32.2 \%$ in patients who received the high-carb diet, but decreased by $24.3 \%$ in the patients on the ketogenic diet $[10,11]$. In another study, three out of five patients on a ketogenic diet combined with radiation or chemotherapy experienced complete treatment. More interesting, the other two participants found the disease progressed after they stopped the ketogenic diet [12].

We had organized a research in the Violet Cancer Institute (VCI) on 54 patients with 6 different types of cancer. The aim of this research is to figure out the effectiveness of the Sorush Cancer Treatment Protocol (SCTP) which is based on the Evolutionary Metabolic Hypothesis of 


\section{Open Access Journal of Mycology \& Mycological Sciences}

Cancer (EMHC) and introducing the Specific Ketogenic Diet (SKD) plus Intravenous Ozone Therapy (IOT) in Phase (1) on 54 cancer patients (Ozone has been found to be an extremely safe medical therapy, free from side effects. In a 1980 study done by the German medical society for ozone therapy, 644 therapists were pulled regarding their 384,775 patients, comprising a total of $5,979,238$ ozone treatments administered. There were only 40 cases of side effects noted out of this number which represents the incredibly low rate of $0.000007 \%$ and only 4 fatalities. Ozone has thus proven to be the safest medical therapy ever devised $[13,14]$, and combination of Hyperbaric Oxygen Therapy with vitamin/ mineral and herbal supplementation beside the SKD and IOT in Phase (2) of this research on the remained 31 cancer patients. Based on the researches from 1928-2016 and the experimentation of cancer treatments and protocols on cancer patients, we have reached a treatment and decided to test it on 54 voluntaries cancer patients in the first stage of their disease. In this treatment we used a 5-day water fasting state, the Specific Ketogenic Diet (SKD) designed by ourselves and Intravenous Ozone Therapy (IOT) in the duration of 90 days (Phase 1) and another 90 days (Phase 2) with the entrance of Hyperbaric Oxygen Therapy (HBO2T) and several supplements which we have been effective in previous studies on cancer patients. We have used the measurement of saliva $\mathrm{PH}$, the MRI device and statistical methods to test the shrinkage of the tumors. After Phase (1) of this research on 54 patients the average percentage decrease in the tumors was 58\% and after Phase (2) on 31 remained cancer patients the average percentage decrease in the tumors was $98.8 \%$. The average saliva $\mathrm{PH}$ in the fasting state of the cancer patients improved from acidic to alkaline as well. In conclusion, we have reached an effective cancer treatment based on SCTP by the usage of SKD, IOT, HBO2T and several supplements. There was an obvious improvement of cancer tumor decrease; lifestyle, saliva $\mathrm{PH}$ and we did not observe any side effects and cachexia in any of the patients [4].

Title: Tumor Size Average Before and After the Protocol.

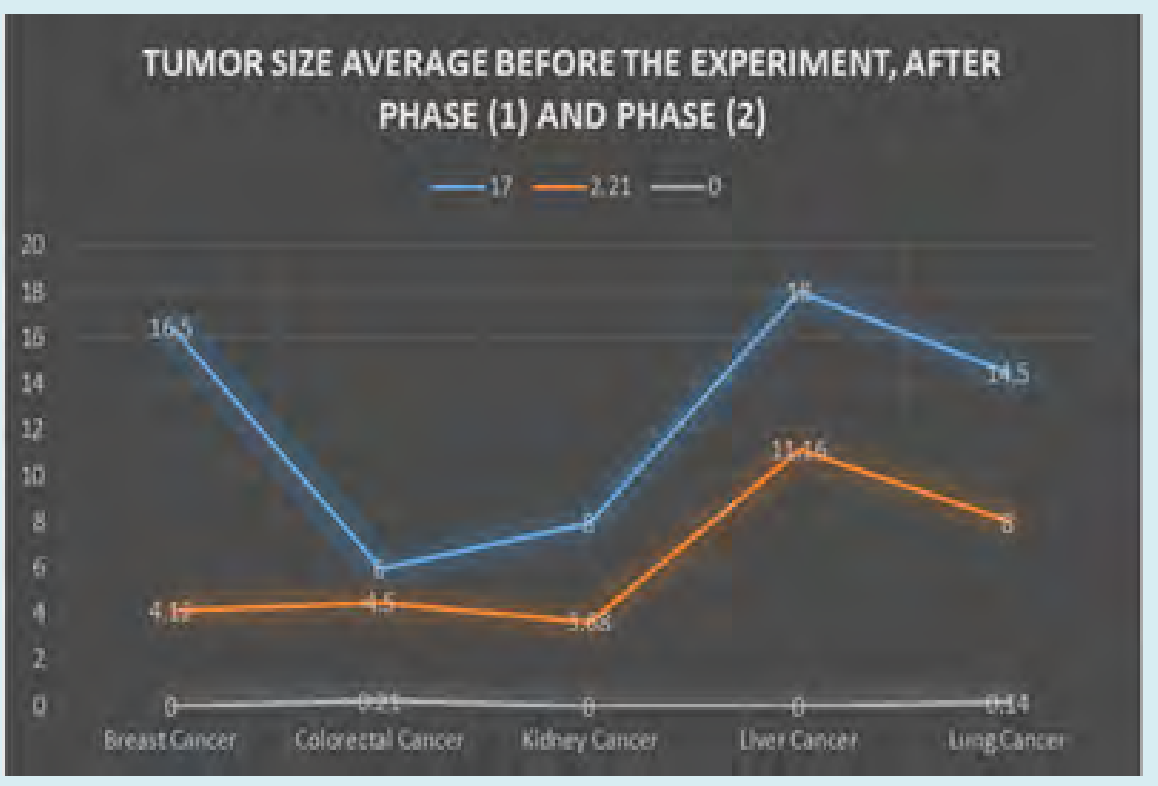

Figure 1: The blue line shows the tumor size before the experiment begins. The orange line shows the tumor size after Phase (1) of the treatment. The white line shows the shrinkage and improvement of the cancer patient's tumors after 180 days.

We named this methodology used in this treatment the Sorush Cancer Treatment Protocol (SCTP) [14]. This protocol includes: 5-day water fasting, SKD, IOT, HBO2T, natural Vitamin/Mineral Supplements, Herbal Supplements, 3000-5000 mg ascorbic acid, probiotic foods and cottage cheese mixed with flaxseed oil. The total calorie intake by the patients should be $1200-1500 \mathrm{Cal} / \mathrm{Day}$. The dietary restrictions in this protocol are: Dairy, Industrial Vegetable oils, Margarines, Alcohol, Gluten and Soy Products (Miso and Natto are exceptional), Artificial Sweeteners as well as Stevia, Fruit juices and any types of sugar including Honey. The duration of the SCTP should not exceed 6 months to reduce the possibilities of ketoacidosis occurrence in patients. 


\section{Open Access Journal of Mycology \& Mycological Sciences}

Title: Sorush Cancer Treatment Protocol in brief.

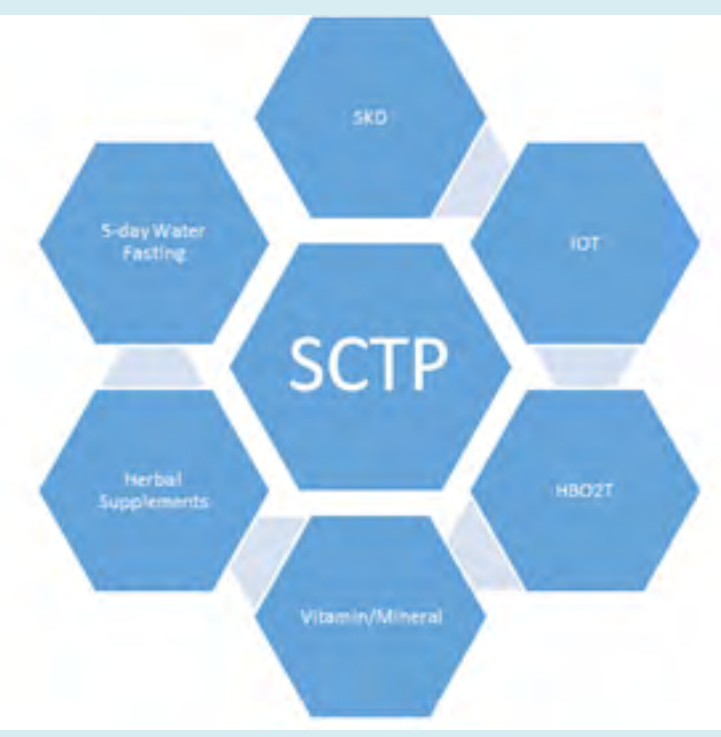

Figure 2: SCTP procedure in brief.

\section{Conclusion}

The most important note in this protocol is that we banned the consumption of industrial vegetable oils and margarine. In our research which we published with the help of Miriam Kalamian EdM, MS, CNS, we concluded that Omega-6 Linoleic acid from vegetable oils increases oxidative stress in the body of humans, contributing to endothelial dysfunction and heart disease. The consumption of these harmful oils which are high in mega- 6 polyunsaturated fats results in changing the structure of cell membrane which contributes to increasing inflammation and the incidence of cancer [15]. We replaced MCT by Extra Virgin Coconut Oil which we found to have more advantages in the Ketogenic Diet for the treatment of cancer [16]. Furthermore; we have prohibited the consumption of any soy products in our protocol since there is many evidences show the relation between the consumption of Soy and cancer incidences specially breast cancer [13].

\section{Acknowledgement}

We would like to mention that the sponsor for all the researches of us, are Weston A. Price Foundation (WAPF) and Violet Cancer Institute (VCI). We would also like to thank specially Professor Thomas N. Seyfried Ph.D. and Professor Stephanie Seneff Ph.D. for their supportive help in of researches, Professor Dominic D'Agostino Ph.D. and Professor Angela Poff Ph.D. for their informative help.

\section{References}

1. Siegel RL, Miller KD, Jemal A (2016) Cancer statistics, 2016. CA Cancer J Clin 66(1): 7-30.

2. Zaminpira S, Niknamian S (2016) Nutritional Ketosis Condition and Specific Ketogenic Diet, May Benefit Cancer Patients as an Alternative Treatment by Sudden Change in the Metabolic State of Cancer Cells. Int J Biochem Physiol 1(2): 000107.

3. Seyfried TN, Huysentruyt LC (2013) On the Origin of Cancer Metastasis. Crit Rev Oncog 18(1-2): 43-73.

4. Zaminpira S, Niknamian S (2017) Evolutionary Metabolic Hypothesis of Cancer (EMHC). EC Pulmonology and Respiratory Medicine 5(4): 167-179.

5. Zaminpira S, Niknamian S (2017) How Butterfly Effect or Deterministic Chaos Theory in Theoretical Physics Explains the Main Cause of Cancer. EC Cancer 2(5): 227238.

6. Volek JS, Fernandez ML, Feinman RD, Phinney SD (2008) Dietary carbohydrate restriction induces a unique metabolic state positively affecting atherogenic dyslipidemia, fatty acid partitioning, and metabolic syndrome. Prog Lipid Res 47(5): 307-318.

7. Allen BG, Bhatia SK, Anderson CM, Eichenberger Gilmore JM, Sibenaller ZA, et al. (2014) Ketogenic diets as an adjuvant cancer therapy: History and potential mechanism. Redox Biol 2: 963-970.

8. Zuccoli G, Marcello N, Pisanello A, Servadei F, Vaccaro $S$, et al. (2010) Metabolic management of glioblastoma multiforme using standard therapy together with a restricted ketogenic diet: Case Report. Nutr Metab (Lond) 7: 33.

9. Nebeling LC, Miraldi F, Shurin SB, Lerner E (1995) Effects of a ketogenic diet on tumor metabolism and nutritional status in pediatric oncology patients: two case reports. J Am Coll Nutr 14(2): 202-208.

10. Schmidt M, Pfetzer N, Schwab M, Strauss I, Kammerer U (2011) Effects of a ketogenic diet on the quality of life in 16 patients with advanced cancer: A pilot trial. Nutr Metab (Lond) 8(1): 54.

11. Fanelli RF, Franchi F, Mulieri M, Cangiano C, Cascino A, et al. (1991) Effect of energy substrate manipulation on tumour cell proliferation in parenterally fed cancer patients. Clin Nutr 10(4): 228-232.

12. Schwartz K, Chang HT, Nikolai M, Pernicone J, Rhee S, et al. (2015) Treatment of glioma patients with ketogenic 
diets: report of two cases treated with an IRB-approved energy-restricted ketogenic diet protocol and review of the literature. Cancer Metab 3: 3.

13. Zaminpira S, Niknamian S (2017) The Effect of Nutrition in the Evolution of Cancer. Royan Pazhouh Publication Group.

14. Zaminpira S, Niknamian S (2017) The Prime Cause and Treatment of Cancer, Lap Lambert Publication Group.
15. Niknamian S, Kalamian M (2016) Vegetable Oils Consumption as One of the Leading Cause of Cancer and Heart Disease. International Science and Investigation Journal 5(5): 1-12.

16. Niknamian S (2016) Dodecanoic-Acid in Extra Virgin Coconut Oil, May Reduce the Incidence of Heart Disease and Cancer in Humans. International Journal of Science and Research 5(11): 792-797. 